Pneumologe 2016 $\cdot 13: 1-3$

DOI 10.1007/s10405-015-0023-0

Online publiziert: 9. Dezember 2015

๑) Springer-Verlag Berlin Heidelberg 2015

CrossMark

\author{
U. Costabel ${ }^{1} \cdot \mathrm{H}$. Wirtz ${ }^{2}$ \\ 'Interstitielle und seltene Lungenkrankheiten, Ruhrlandklinik, Westdeutsches Lungenzentrum, \\ Universitätsklinikum Essen, Essen, Deutschland \\ ${ }^{2}$ Abteilung Pneumologie, Universitätsklinikum Leipzig, Leipzig, Deutschland
}

\title{
Seltene Lungenkrankheiten
}

Dieses Themenheft erscheint anlässlich des diesjährigen DGP-Kongresses, der in Leipzig unter der Präsidentschaft von $H$. Wirtz stattfinden wird. Es ist den seltenen Lungenerkrankungen gewidmet. Auf vielen früheren DGP-Kongressen stießen Symposien zu diesem Thema stets auf größtes Interesse. Das liegt einerseits daran, dass Kenntnisse über Krankheiten mit einer Inzidenz von 1-5 auf 100.000 Personen naturgemäß kein medizinisches Allgemeingut sind, andererseits gerade hier große Fortschritte in Grundlagenwissenschaft und klinischem Management erzielt wurden. Das vorliegende Heft soll einige dieser Entwicklungen aufgreifen und dadurch die langjährigen Bemühungen der beiden Herausgeber unterstützen, das Wissen über seltene Erkrankungen zum Wohl der betroffenen Patienten zu verbreiten. Wir haben für dieses Heft „Klassiker“ der seltenen interstitiellen Lungenerkrankungen sowie Krankheiten aus dem pädiatrischen, vaskulären und onkologischen Bereich ausgewählt.

\section{\) Das Wissen über seltene Lungenkrankheiten ist wertvoll}

Im ersten Beitrag über die pulmonale Alveolarproteinose (PAP) gehen F. Bonella und $U$. Costabel auf die faszinierenden neuen Erkenntnisse zu dieser seltenen Krankheit ein. Nach pathogenetischen Mechanismen werden heute die primären Formen (autoimmun oder hereditär) mit gestörter GM-CSF-Signaltransduktion von den sekundären, bei denen die Alveolarmakrophagen in Zahl und Funktion reduziert sind, sowie von den alveolarproteinose-ähnlichen Syndromen abgegrenzt, bei denen Genmutationen zu gestörten Surfactant- produktions- und/oder Transportmechanismen führen. Die häufigste Form der PAP, die primäre autoimmune, kann durch den Nachweis von GM-CSF-Antikörpern im Serum der betroffenen Patienten sicher von anderen Entitäten abgegrenzt werden. Während die Ganzlungenlavage unverändert die Therapie der ersten Wahl ist, warten wir gespannt auf die Ergebnisse randomisierter Studien, die zeigen werden, ob inhalative GM-CSF-Substitution oder Rituximab zur neuen Standardtherapie werden.

Der zweite Beitrag von $H$. Wirtz befasst sich mit neuen Therapieansätzen bei der Lymphangioleiomyomatose (LAM). Auch bei dieser seltenen Erkrankung haben Erkenntnisse der Grundlagenforschung, z. B. die Verursachung der sporadischen LAM durch eine Mosaikmutation des TSC2-Gens, was Gemeinsamkeiten mit der tuberösen Sklerose erklärt, unmittelbare Konsequenzen für die Therapie nach sich gezogen. Bei Mutation des TSC2-Gens wird Tuberin, ein Tumorsuppressorgen, nicht ausreichend zur Verfügung gestellt. Hier greift der Mechanismus von Rapamycin (Sirolimus) ein: Es kann ähnlich wie Tuberin mTOR inhibieren und so die überschießende Proliferation, z. B. der LAM-Zellen, reduzieren. Die pathophysiologischen Vorgänge sind naturgemäß ungleich komplexer, mit zahlreichen weiteren beteiligten Signalwegen, die durch Medikamente eventuell beeinflussbar sind, wie es ausführlich und spannend in diesem Artikel beschrieben wird. Neben Rapamycin hat sich kürzlich Erolimus als weiterer mTOR-Antagonist in einer placebokontrollierten Studie als wirksam erwiesen. Auch ein neuer diagnostischer Aspekt sollte nicht unerwähnt bleiben: VEGF-D ist im Serum 
von LAM-Patientinnen erhöht, sodass dies als diagnostisches Merkmal zur Abgrenzung von anderen Erkrankungen mit Lungenzysten klinisch genutzt werden kann.

Im dritten Beitrag gibt $H$. Olschewski einen hervorragenden Überblick über die pulmonale veno-okklusive Erkrankung (PVOD). Sie ist eine seltene Differenzialdiagnose zur idiopathischen und zur hereditären pulmonal-arteriellen Hypertonie, spricht aber deutlich schlechter auf (PAH)-Medikation an und neigt unter Therapie zur Hypoxämie und zum Lungenödem. Das wichtigste diagnostische Merkmal sind Veränderungen im Dünnschicht-CT der Lunge mit multiplen milchglasartigen Verdichtungen und verdickten interlobulären Septen, in der BAL finden sich Hinweise auf ein alveoläres Hämorrhagiesyndrom. Die Ätiologie ist noch weitgehend unklar, es häufen sich jedoch Beobachtungen, welche die Bedeutung genetischer Faktoren unterstreichen. Zunehmend werden familiäre Fälle mit nachgewiesenen $\mathrm{Mu}$ tationen bestimmter Gene bekannt.

Im vierten Beitrag beschreiben $C$. Werner und H. Omran das aktuelle Wissen zur primären ziliären Dyskinesie (PCD), einer seltenen autosomal rezessiv vererbten Erkrankung der Struktur oder Funktion motiler Zilien. Da die PCD genetisch sehr heterogen ist, existieren zahlreiche unterschiedliche PCD-Varianten. Die Diagnostik erfordert den Einsatz unterschiedlicher Verfahren. Die Hochfrequenz-Videomikroskopie (HVNA) erlaubt eine direktmikroskopische Analyse des Zilienschlages, der Zilienschlagfrequenz und des Schlagmusters, während die hochauflösende immunfluoreszenzmikroskopische Untersuchung eine detaillierte Strukturanalyse der Zilien ermöglicht. H. Omran und seiner Arbeitsgruppe gelang es in den letzten Jahren, eine Vielzahl neuer Genmutationen nachzuweisen, sodass heute in diesem Expertenzentrum etwa $60 \%$ der Fälle mittels genetischer Verfahren aufgeklärt werden können.

Im letzten Beitrag stellt A. Frille ausgewählte seltene thorakale Tumorerkrankungen vor. Alle seltenen thorakalen Tumore zusammen umfassen überraschenderweise ungefähr $22 \%$ aller Krebsdia- gnosen und $21 \%$ aller thorakalen Tumoren. Gerade für die zahlreichen seltenen thorakalen Tumoren ist es wichtig, dass sie in pneumologisch-onkologischen Kompetenzzentren behandelt werden und an international kooperierende Krebsregister angebunden werden. Anders sind neue Erkenntnisse zur Therapie und Prognose, beispielsweise der diffusen idiopathischen pulmonalen neuroendokrinen Zellhyperplasie, des adenoidzystischen Karzinoms, des lokalisierten malignen Mesothelioms oder des Thymuskarzinoms, kaum zu erzielen.

Schließlich möchten wir uns bei allen Autoren für ihre gelungenen Beiträge bedanken. Den Lesern wünschen wir, dass möglichst vielen nach der Lektüre dieses Heftes die Diagnose einer seltenen Lungenerkrankung gelingen wird und sie durch das zauberhafte Gefühl beglückt werden, was damit verbunden ist.

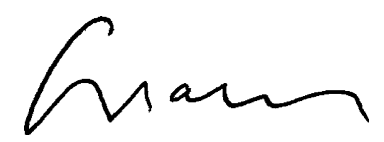

Prof. Dr. Ulrich Costabel

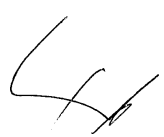

Prof. Dr. Hubert Wirtz

\section{Korrespondenzadresse}

\section{Prof. Dr. U. Costabel}

Interstitielle und seltene Lungenkrankheiten, Ruhrlandklinik, Westdeutsches Lungenzentrum, Universitätsklinikum Essen Tüschener Weg 40, 45239 Essen, Deutschland Ulrich.Costabel@ruhrlandklinik.uk-essen.de

Interessenkonflikt. U. Costabel und H. Wirtz geben an, dass kein Interessenkonflikt besteht.

\section{Entwicklung von Kindern und Jugendlichen}

KiGGS: Welle 1

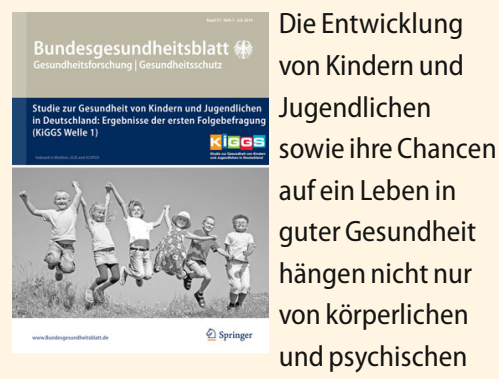

Faktoren ab, sondern werden auch durch das familiäre und soziale Umfeld geprägt. Diese Zusammenhänge untersucht die erste Folgeerhebung (KiGGS Welle 1) der "Studie zur Gesundheit von Kindern und Jugendlichen in Deutschland" (KiGGS). Im Schwerpunktheft "Studie zur Gesundheit von Kindern und Jugendlichen in Deutschland: Ergebnisse der ersten Folgebefragung (KiGGS: Welle 1)" (Ausgabe 7/2014) der Zeitschrift Bundesgesundheitsblatt werden u.a. folgende wichtige Daten, Analysen und Trendentwicklungen aus der neuen KiGGS-Befragungswelle vorgestellt:

- Chronische Erkrankungen und impfpräventable Infektionserkrankungen

- Tabak- und Alkoholkonsum bei 11-bis 17-jährigen Jugendlichen

- Einflussfaktoren auf Verbreitung und Dauer des Stillens in Deutschland

- Körperlich-sportliche Aktivität und Nutzung elektronischer Medien im Kindesund Jugendalter

Bestellen Sie diese Ausgabe zum Preis von 16,--EUR zzgl. Versandkosten bei Springer Customer Service Center Kundenservice Zeitschriften

Haberstr. 7

69126 Heidelberg

Tel.: +49 6221-345-4303

Fax: +49 6221-345-4229

E-Mail: leserservice@springer.com

Suchen Sie noch mehr zum Thema? Mit e.Med, dem Online-Paket von Springer Medizin, können Sie schnell und komfortabel in über 600 medizinischen Fachzeitschriften recherchieren. Weitere Infos unter springermedizin.de/eMed. 
Hier steht eine Anzeige.

算 Springer 